\title{
Influência de Diferentes Concentrações de Vermicomposto no Desenvolvimento de Mudas de Eucalipto e Pinus
}

\author{
Rodrigo Ferreira da Silva ${ }^{1}$, Rudinei De $\mathrm{Marco}^{1}$, Clovis Orlando Da Ros ${ }^{1}$, \\ Hazael Soranzo de Almeida ${ }^{1}$, Zaida Inês Antoniolli ${ }^{2}$ \\ ${ }^{1}$ Departamento de Ciências Agronômicas e Ambientais, Universidade Federal de Santa Maria - UFSM, \\ Frederico Westphalen/RS, Brasil \\ ${ }^{2}$ Departamento de Solos, Universidade Federal de Santa Maria - UFSM, Santa Maria/RS, Brasil
}

\begin{abstract}
RESUMO
O objetivo do estudo foi avaliar o efeito da vermicompostagem de resíduos de carne e esterco bovino no desenvolvimento de mudas de eucalipto e pinus. O experimento foi conduzido em delineamento inteiramente casualizado com oito tratamentos, sendo quatro elaborados a partir da adição de $0 \%, 20 \%, 40 \%$ e $60 \%$ de esterco bovino ao resíduo de carne, ambos vermicompostados, e quatro constituídos pela adição de $0 \%, 20 \%, 40 \%$ e $60 \%$ de vermicomposto ao substrato comercial, com sete repetições. Foram avaliados a altura das mudas, o diâmetro do colo, a massa seca da parte aérea e radicular, a área superficial específica, o índice de qualidade de Dickson e a relação entre a altura da muda e o diâmetro do colo. A adição ao substrato comercial de $20 \%$ e $40 \%$ de vermicomposto proporciona desenvolvimento e qualidade de mudas de eucalipto equivalentes ao alcançado com a utilização de $100 \%$ de substrato comercial. As mudas de pinus não são favorecidas pelas proporções de vermicomposto testadas.
\end{abstract}

Palavras-chave: esterco bovino, resíduo orgânico, espécies florestais, Eucalyptus grandis, Pinus elliottii.

\section{Influence of Different Concentrations of Vermicompost in the Development of Eucalyptus and Pine Seedlings}

\begin{abstract}
The objective of this study was to evaluate the effect of meat waste and cattle manure vermicomposting in the development of eucalyptus and Pinus seedlings. The experiment was conducted in a completely randomized design with eight treatments with seven repetitions. The treatments were: $0 \%, 20 \%, 40 \%$ and $60 \%$ of cattle manure added to meat waste, both vermicomposted, and $0 \%, 20 \%, 40 \%$ and $60 \%$ of the vermicompost added to commercial substrate. Stem height and diameter, shoot and root dry mass, specific surface area, Dickson quality index and the ratio between stem height and diameter were assessed. Addition of $20 \%$ and $40 \%$ of vermicompost in eucalyptus seedling cultivation promotes similar development and quality obtained with addition of $100 \%$ of commercial substrate. Pinus seedlings are not influenced by the proportions of vermicompost tested.
\end{abstract}

Keywords: cattle manure, organic waste, forest species, Eucalyptus grandis, Pinus elliottii. 


\section{INTRODUÇÃO}

Os resíduos orgânicos, principalmente os de processamento de produtos, como o abate de animais, podem acarretar problemas ao meio ambiente quando destinados de forma inadequada (Edvan \& Carneiro, 2011), especialmente pelo elevado conteúdo de matéria orgânica e pela carga microbiológica que o mesmo apresenta (Pardi et al., 2006). Diante disso, Barros \& Licco (2007) relataram que os resíduos de origem animal podem ser destinados basicamente para a incineração ou a reciclagem, sendo a reciclagem considerada a via de destinação mais adequada, em razão do aspecto ambiental e da saúde pública e financeira.

Trabalhos de pesquisa têm demonstrado que a vermicompostagem tem grande potencial para reciclagem de resíduos orgânicos, minimizando os problemas ocasionados pelo descarte inadequado dos resíduos no meio ambiente. A vermicompostagem resulta em um produto considerado adubo orgânico, podendo substituir a utilização de adubação mineral (Steffen et al., 2011; Antunes et al., 2016).

O vermicomposto é produzido com auxílio de minhocas e apresenta, como produto final, um composto com elevado valor nutricional, contendo fósforo, cálcio e potássio, que podem ser utilizados na composição de substratos para a produção de mudas de espécies florestais (Araújo et al., 2009; Steffen et al., 2011). Desse modo, a produção de vermicomposto pode ser uma alternativa para proporcionar nutrientes e condições de crescimento aos substratos utilizados para produção de mudas.

De acordo com a ABRAF (2012), o Rio Grande do Sul possui a quinta maior área de plantio de espécies do gênero Eucalyptus, com 280.198 ha, e a terceira maior área do gênero Pinus, com 164.806 ha, dentre os Estados brasileiros. Contudo, constantemente se busca melhorar a produtividade das plantações florestais. Nesse contexto, Sarzi et al. (2010) relatam que uma das principais fases para o estabelecimento de bons povoamentos florestais é a produção de mudas de qualidade, sendo, nesse caso, de fundamental importância a escolha de um substrato adequado para possibilitar o desenvolvimento de mudas de qualidade.

Aliado a isso, trabalhos apresentados na literatura evidenciam o efeito benéfico do composto ou do vermicomposto proveniente de diferentes resíduos orgânicos como substrato para a produção de mudas florestais (Silva et al., 2014; Vieira et al., 2014).

Neste sentido, o objetivo do estudo foi avaliar o efeito da vermicompostagem de resíduos de carne e esterco bovino no desenvolvimento e na qualidade de mudas de Eucalyptus grandis e Pinus elliottii.

\section{MATERIAL E MÉTODOS}

O estudo foi desenvolvido em casa de vegetação de plástico não climatizada, pertencente à Universidade Federal de Santa Maria, Campus Frederico Westphalen, RS, situado na latitude de $27^{\circ} 23^{\prime} 47^{\prime \prime}$ Sul e longitude de $53^{\circ} 25^{\prime} 41^{\prime \prime}$ Oeste, a uma altitude média de $465 \mathrm{~m}$, com duas espécies florestais, caracterizando dois experimentos: um com eucalipto (Eucalyptus grandis Hill ex Maiden) e outro com Pinus (Pinus elliottii var. elliottii Engelm).

O delineamento experimental utilizado para ambos os experimentos foi inteiramente casualizado com oito tratamentos, sendo quatro elaborados a partir da adição de $0 \%, 20 \%, 40 \%$ e $60 \%$ de esterco de bovino (EB) ao resíduo de carne (RC), ambos vermicompostados (por um período de 60 dias), e quatro constituídos pela adição de $0 \%, 20 \%, 40 \%$ e $60 \%$ de vermicomposto (VC) ao substrato comercial (SC) Plantmax ${ }^{\circ}$. Cada tratamento foi composto por sete repetições e cada repetição foi composta por uma muda por tubete (Tabela 1).

O vermicomposto utilizado para a formulação dos tratamentos com o substrato comercial foi na proporção RC 40\%:EB 60\% [a utilização deste substrato deve-se a resultados preliminares que indicaram elevado potencial para a produção de mudas e para a multiplicação de Oligochaetas (dados não publicados)]. O resíduo de carne submetido à vermicompostagem era constituído de óleos e graxas de origem suína, resíduos da queima de madeira, hidróxido de cálcio e serragem. O esterco bovino foi oriundo da criação de gado de corte. Para a vermicompostagem foram utilizados recipientes de polipropileno com capacidade de $50 \mathrm{~L}$, sendo os constituintes misturados uniformemente, de acordo com as proporções dos tratamentos. Após 20 dias de estabilização (compostagem), quando a temperatura do composto reduziu para $25^{\circ} \mathrm{C}$, foram adicionadas 1.000 minhocas da espécie Eisenia andrei, que efetuaram a vermicompostagem durante 60 dias. O material foi 
Tabela 1. Tratamentos utilizados no trabalho e sua respectiva caracterização química, pH, N-mineral, N, $\mathrm{P}$ e K, para a produção de mudas de Eucalyptus grandis e Pinus elliottii.

Table 1. Treatments used in the study and their respective chemical characterization, $\mathrm{pH}$, mineral N, N, P and K for the production of seedlings of Eucalyptus grandis and Pinus elliottii.

\begin{tabular}{|c|c|c|c|c|c|c|}
\hline \multirow{3}{*}{ Tratamentos } & \multirow{3}{*}{$\mathrm{pH} \mathrm{em} \mathrm{H}_{2} \mathrm{O}$} & \multicolumn{2}{|c|}{$\mathrm{N}$-mineral } & \multicolumn{3}{|c|}{ Nutrientes Totais } \\
\hline & & $\mathbf{N}-\mathrm{NH}_{4}^{+}$ & $\mathrm{N}-\mathrm{NO}_{3}$ & $\mathbf{N}$ & $\mathbf{P}$ & $\mathbf{K}$ \\
\hline & & \multicolumn{2}{|c|}{---------- $\mathrm{mg} \mathrm{kg}^{-1}$--------- } & ----- & $\mathrm{kg}$ & --- \\
\hline RC 100\%:EB 0\% & 8,60 & 62,1 & 385,0 & 4,08 & 1,3 & 4,3 \\
\hline RC $80 \%$ :EB $20 \%$ & 8,40 & 62,4 & $1.052,2$ & 8,65 & 1,6 & 9,8 \\
\hline RC 60\%:EB 40\% & 7,70 & 113,3 & $1.744,6$ & 6,18 & 1,5 & 5,4 \\
\hline RC $40 \%: E B \quad 60 \%$ & 7,50 & 136,3 & $1.962,8$ & 7,14 & 1,3 & 6,3 \\
\hline SC $100 \%: V C 0 \%$ & 7,00 & 134,8 & 70,7 & 5,22 & 0,7 & 4,5 \\
\hline SC $80 \%: V C ~ 20 \%$ & 7,60 & 110,1 & 749,7 & 6,10 & 0,9 & 5,0 \\
\hline SC $60 \%: \mathrm{VC} 40 \%$ & 7,60 & 133,2 & $1.223,8$ & 5,68 & 1,0 & 4,9 \\
\hline SC $40 \%: \mathrm{VC} 60 \%$ & 7,50 & 118,9 & $1.441,7$ & 6,36 & 1,1 & 5,3 \\
\hline
\end{tabular}

$\mathrm{RC}=$ resíduo de carne; $\mathrm{SC}=$ substrato comercial; $\mathrm{EB}=$ esterco bovino; $\mathrm{VC}=$ vermicomposto (RC 40\%:EB 60\%).

revolvido com auxílio de um rastelo e umedecido semanalmente.

De uma amostra de cada tratamento, foram extraídos os teores totais de N, P e K por meio de digestão com ácido sulfúrico, sendo quantificados os seus teores por destilação e titulação $(\mathrm{N})$, por espectrofotometria $(\mathrm{P})$ e por fotometria de chamas (K). Os teores de $\mathrm{N}$ mineral foram extraídos com solução $\mathrm{KCl} 1 \mathrm{~mol} \mathrm{~L}^{-1}$, sendo quantificados por destilação e titulação os teores de

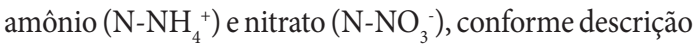
em Tedesco et al. (1995). Os teores encontrados em cada tratamento estão especificados na Tabela 1.

As sementes das espécies em estudo (Eucalyptus grandis e Pinus elliottii var. elliottii) foram oriundas do banco de sementes da Estação de Pesquisa Florestais do Rio Grande do Sul, FEPAGRO - Floresta, Santa Maria, RS. No mês de março, foi realizada a semeadura em tubetes de polipropileno com capacidade de $125 \mathrm{~cm}^{3}$ a $0,5 \mathrm{~cm}$ de profundidade, de modo a evitar a dessecação das sementes, utilizando-se três destas por tubete. Decorridos 30 dias da semeadura, foi procedido um desbaste, permanecendo apenas uma muda por tubete, considerando-se os aspectos de fitossanidade e vigor. Durante o período de condução dos experimentos, a irrigação foi realizada por microaspersores, acionados por time, no início da manhã, às $8 \mathrm{~h}$, e a última, às $18 \mathrm{~h}$, tendo duração de 12 minutos cada irrigação e com intervalos entre irrigações de $2 \mathrm{~h}$.

O experimento foi conduzido por 120 dias para o eucalipto e 180 dias para o pinus. Ao final desse período, foram avaliados: a altura das mudas $(\mathrm{H})$, o diâmetro do colo (DC), a massa seca da parte aérea (MSPA), a massa seca radicular (MSR) e a área superficial específica radicular (ASE). A altura das mudas foi medida a partir da região do colo da muda até o ápice da haste principal, com o auxílio de uma régua graduada, e o diâmetro do colo, com um paquímetro digital (Digimess ${ }^{\circledR}$ ). Para a determinação da MSPA e da MSR, separou-se o sistema radicular da parte aérea, na região do colo da muda. Na sequência, o sistema radicular foi lavado com jatos de água sobre peneiras com malha de $1 \mathrm{~mm}$. Posteriormente, as partes aérea e radicular foram mantidas em estufa de circulação forçada de ar a $65 \pm 1{ }^{\circ} \mathrm{C}$, até peso constante, sendo, então, pesadas em balança analítica. A área superficial específica radicular foi estimada conforme o método de Tennant (1975), por meio da Equação 1:

$\mathrm{ASE}=2 \pi \times \mathrm{RR} \times \mathrm{L}$

em que: ASE = área superficial específica; $\mathrm{L}=$ comprimento radicular; $\mathrm{RR}=$ raio de raízes.

Para o cálculo do raio, utilizou-se a Equação 2:

$\mathrm{RR}=\frac{\sqrt{\mathrm{V}}}{\mathrm{L} \times \pi}$

em que: $\mathrm{V}=$ volume (massa verde do sistema radicular).

O comprimento radicular foi estimado conforme Tennant (1975), utilizando a Equação 3:

$\mathrm{L}=\mathrm{NI} \times \mathrm{Fc}$

Em que: $\mathrm{L}=$ comprimento do sistema radicular; $\mathrm{NI}=$ número de intersecções. 
Para essa estimativa, separou-se uma amostra de 0,2 g de raízes cortadas em $1 \mathrm{~cm}$, e distribuiu-se em uma placa de Petri quadriculada de $1 \mathrm{~cm} \times 1 \mathrm{~cm}$. Em seguida, contou-se o número de intersecções das raízes com as linhas da placa e Fc (Fator de correção) = 0,7857.

A partir dos parâmetros morfológicos avaliados, foram calculados os índices de qualidade de mudas, com base na relação entre: altura da muda e diâmetro do colo (H/DC), e o índice de qualidade de Dickson, proposto por Dickson et al. (1960), por meio da Equação 4:

$\mathrm{IQD}=\frac{\mathrm{MSPA}+\mathrm{MSR}}{\frac{\mathrm{H}}{\mathrm{DC}}+\frac{\mathrm{MSPA}}{\mathrm{MSR}}}$

A eficiência relativa (ER) dos tratamentos propostos (TP), constituídos de proporções de vermicompostos de resíduos orgânicos, em relação ao tratamento 100\% substrato comercial (SC), foi calculada de acordo com a Equação 5:

$\mathrm{ER}(\%)=\frac{\mathrm{SC}-\mathrm{TP}}{\mathrm{SC}} \times-100$

Para atender aos pressupostos da análise de variância, realizaram-se os testes de normalidade e homogeneidade das variâncias, pelos testes de Shapiro-Wilk e Bartlett, respectivamente. Foi utilizada a Equação $\sqrt{(\mathrm{x}+1)}$, quando houve a necessidade de transformação dos dados (variáveis MSR e ASE). Atendidas as pressuposições matemáticas, procedeu-se à análise de variância pelo teste $\mathrm{F}$ e as médias foram comparadas pelo teste de Tukey, a 5\% de probabilidade de erro, utilizando o programa estatístico SISVAR (Ferreira, 2016).

\section{RESULTADOS E DISCUSSÃO}

As mudas de Eucalyptus grandis evidenciaram significativamente $(\mathrm{p} \leq 0,05)$ maior desenvolvimento em altura, diâmetro do colo e massa seca da parte aérea no tratamento $100 \%$ de substrato comercial e nos tratamentos constituídos pela mistura em diferentes proporções do substrato comercial com o vermicomposto (Tabela 2). Steffen et al. (2011) relataram que a proporção de $80 \%$ de vermicomposto de esterco bovino criado em confinamento e $20 \%$ de turfa proporcionam maior desenvolvimento em mudas de E. grandis, enquanto que, para a produção de mudas de Corymbia citriodora, o maior crescimento ocorreu em proporções que variaram de $40 \%$ a $80 \%$ de vermicomposto. Os resultados do presente estudo reforçam os encontrados por Steffen et al. (2011), em que a combinação de vermicomposto e substrato comercial proporcionam desenvolvimento similar ao uso de somente substrato comercial para as mudas de eucaliptos.

O tratamento contendo $80 \%$ de substrato comercial com adição de $20 \%$ de vermicomposto proporcionou maior massa seca radicular para as mudas de E. grandis, não diferindo significativamente $(\mathrm{p} \leq 0,05)$ dos tratamentos com $100 \%$ de substrato comercial e da proporção $60 \%$ substrato comercial mais $40 \%$ de vermicomposto (Tabela 2). Em estudos semelhantes realizados por Steffen et al. (2011), o tratamento com $80 \%$ de vermicomposto de esterco bovino mais $20 \%$ de turfa proporcionou maior massa seca radicular nas mudas de E. grandis. Schumacher et al. (2001), também estudando o efeito da vermicompostagem no desenvolvimento de mudas de E. grandis, observaram que o aumento da massa seca radicular foi proporcional ao aumento nas doses de vermicomposto. Possivelmente, esta diferença entre os resultados da literatura para a massa seca radicular do E. grandis esteja relacionada ao nível de estabilidade dos substratos e à quantidade de nutrientes disponíveis para as mudas, podendo estimular a expansão radicular em caso de baixa disponibilidade de nutrientes, conforme relatado por Zhang \& Forde (2000) e Edwards (2004).

A maior área superficial específica radicular (ASE) das mudas de E. grandis foi obtida com $100 \%$ de substrato comercial, diferenciando significativamente $(\mathrm{p} \leq 0,05)$ apenas do substrato $100 \%$ resíduo de carne vermicompostado (Tabela 2). De acordo com Silva et al. (2011), a ASE é um parâmetro de grande importância para a obtenção do sistema radicular com maior capacidade de absorção de nutrientes, uma vez que, para um mesmo peso de raízes, aquela que apresentar maior área superficial específica radicular irá possuir maior quantidade de raízes finas (Tennant, 1975) e, consequentemente, maior capacidade de absorção de nutrientes. Portanto, os resultados deste trabalho demonstram que a utilização de substratos vermicompostados pode contribuir equivalentemente à utilização de $100 \%$ de substrato comercial, para a área superficial específica radicular das mudas de E. grandis. 
Tabela 2. Médias de altura (H), diâmetro do colo (DC), massa seca da parte aérea (MSPA), massa seca radicular (MSR) e área superficial específica radicular (ASE) de mudas de Eucalyptus grandis e Pinus elliottii, produzidas em diferentes proporções de substratos orgânicos vermicompostados.

Table 2. Average height (H), stem diameter (DC), shoot dry mass (MSPA), root dry mass (MSR) and specific surface area of roots (ASE) seedlings of Eucalyptus grandis and Pinus elliottii, produced in different proportions of organic substrate vermicomposted.

\begin{tabular}{|c|c|c|c|c|c|}
\hline \multirow{2}{*}{ Tratamentos } & $\mathbf{H}(\mathbf{c m})$ & $\mathrm{DC}(\mathbf{m m})$ & MSPA (g) & $\operatorname{MSR}(\mathbf{g})$ & $\operatorname{ASE}\left(\mathrm{cm}^{2}\right)$ \\
\hline & \multicolumn{5}{|c|}{ 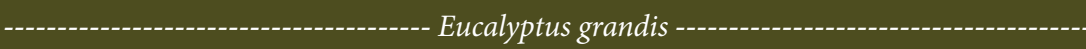 } \\
\hline RC 100\%:EB 0\% & $5,76 b^{*}$ & $0,79 \mathrm{e}$ & $0,03 \mathrm{c}$ & $0,03 \mathrm{c}$ & $15,2 \mathrm{~b}$ \\
\hline RC $80 \%: E B ~ 20 \%$ & $7,80 \mathrm{~b}$ & $1,58 \mathrm{~d}$ & $0,28 \mathrm{~b}$ & $0,25 \mathrm{c}$ & $23,2 \mathrm{ab}$ \\
\hline RC 60\%:EB 40\% & $6,32 \mathrm{~b}$ & $1,82 \mathrm{bcd}$ & $0,26 \mathrm{~b}$ & $0,27 \mathrm{c}$ & $22,4 \mathrm{ab}$ \\
\hline RC 40\%:EB 60\% & $7,12 \mathrm{~b}$ & $1,72 \mathrm{~cd}$ & $0,26 \mathrm{~b}$ & $0,26 c$ & $23,5 \mathrm{~b}$ \\
\hline SC 100\%:VC 0\% & $15,34 \mathrm{a}$ & $2,08 \mathrm{abc}$ & $0,39 \mathrm{ab}$ & $0,43 \mathrm{~b}$ & $31,3 \mathrm{a}$ \\
\hline SC $80 \%: V C ~ 20 \%$ & $17,80 \mathrm{a}$ & $2,45 \mathrm{a}$ & $0,45 \mathrm{a}$ & $0,51 \mathrm{a}$ & $26,8 \mathrm{a}$ \\
\hline SC 60\%:VC 40\% & $18,60 \mathrm{a}$ & $2,25 \mathrm{ab}$ & $0,48 \mathrm{a}$ & $0,43 \mathrm{~b}$ & $26,1 \mathrm{a}$ \\
\hline SC $40 \%: V C 60 \%$ & $17,51 \mathrm{a}$ & $2,17 \mathrm{abc}$ & $0,47 \mathrm{a}$ & $0,36 \mathrm{bc}$ & $28,5 \mathrm{a}$ \\
\hline \multirow[t]{2}{*}{$\mathrm{CV} \%$} & 6,90 & 5,38 & 3,08 & 2,6 & 5,38 \\
\hline & $9,20 \mathrm{~b}$ & $1,98 \mathrm{c}$ & $\begin{array}{l}\text { nus ellio } \\
0,18 \mathrm{~d}\end{array}$ & $0,18 c$ & $78,9 \mathrm{~d}$ \\
\hline RC $80 \%: E B ~ 20 \%$ & $12,70 \mathrm{ab}$ & $2,09 \mathrm{c}$ & $0,20 \mathrm{~cd}$ & $0,16 \mathrm{c}$ & $84,3 \mathrm{~d}$ \\
\hline RC 60\%:EB 40\% & $12,10 \mathrm{ab}$ & $2,25 \mathrm{bc}$ & $0,32 \mathrm{bc}$ & $0,24 \mathrm{c}$ & $80,9 \mathrm{~d}$ \\
\hline RC 40\%:EB 60\% & $11,20 \mathrm{ab}$ & $2,16 \mathrm{c}$ & $0,25 \mathrm{~cd}$ & $0,19 c$ & $106,4 \mathrm{~cd}$ \\
\hline SC 100\%:VC 0\% & $14,67 \mathrm{a}$ & $3,15 \mathrm{a}$ & $0,61 \mathrm{a}$ & $0,62 \mathrm{a}$ & $353,9 \mathrm{a}$ \\
\hline SC $80 \%$ :VC $20 \%$ & $12,78 \mathrm{ab}$ & $2,91 \mathrm{ab}$ & $0,45 \mathrm{ab}$ & $0,45 \mathrm{~b}$ & $226,5 \mathrm{~b}$ \\
\hline SC 60\%:VC 40\% & $12,30 \mathrm{ab}$ & $2,50 \mathrm{~b}$ & $0,33 \mathrm{c}$ & $0,32 \mathrm{bc}$ & $165,4 \mathrm{bc}$ \\
\hline SC $40 \%: V C 60 \%$ & $12,60 \mathrm{ab}$ & $2,64 \mathrm{ab}$ & $0,34 \mathrm{bc}$ & $0,25 \mathrm{c}$ & $111,9 \mathrm{~cd}$ \\
\hline $\mathrm{CV} \%$ & 5,76 & 6,54 & 4,48 & 5,42 & 15,27 \\
\hline
\end{tabular}

$\mathrm{RC}=$ resíduo de carne; $\mathrm{EB}=$ esterco bovino; $\mathrm{VC}=$ vermicomposto $(\mathrm{RC} 40 \%: \mathrm{EB} 60 \%) ; \mathrm{SC}=$ substrato comercial. ${ }^{*}$ Médias seguidas de mesma letra na coluna não diferiram estatisticamente a $5 \%$ de probabilidade de erro pelo teste de Tukey.

A maior altura das mudas de Pinus elliottii foi obtida com $100 \%$ de substrato comercial $(14,67 \mathrm{~cm})$, não diferindo $(\mathrm{p} \leq 0,05)$ dos tratamentos com proporções de vermicomposto (Tabela 2). Caldeira et al. (2003) afirmaram que as mudas de $P$. elliottii respondem de modo significativo às doses de vermicomposto e que a utilização de $20 \%$ de vermicomposto proporcionou a maior altura, com $3,6 \mathrm{~cm}$ superiores à altura da muda testemunha (somente solo). Conforme Lambers \& Poorter (2004), espécies de crescimento lento, como é o caso do pinus em relação ao eucalipto, são menos responsivas ao fornecimento de nutrientes. Desse modo, é possível que essa resposta do P. elliottii tenha impossibilitado diferença significativa na altura das mudas obtidas neste trabalho, com a utilização dos tratamentos com proporções de vermicomposto que, consequentemente, possui maior concentração de nutrientes que o substrato comercial, conforme descrito na caracterização química dos substratos (Tabela 1).
Os tratamentos com $100 \%$ de substrato comercial e os com proporções de vermicomposto com substrato comercial proporcionam maior diâmetro do colo nas mudas de $P$. elliottii, em relação aos demais tratamentos (Tabela 2). A haste e a região do colo espesso indicam a presença de substâncias de reserva nos tecidos internos da planta, sendo indicativo que a muda apresenta aspecto sadio e está nutricionalmente apta para o plantio a campo, pois parte das reservas para formar novas raízes vem de nutrientes contidos na haste (Scremin-Dias et al., 2006). Neste sentido, por proporcionar a maior média de diâmetro do colo, a utilização de $100 \%$ de substrato comercial pode ser um indicativo de que as mudas de P. elliottii estão em melhor condição para o plantio a campo.

A utilização de $100 \%$ de substrato comercial proporcionou a maior média da massa seca da parte aérea das mudas de $P$. elliottii, não diferindo $(\mathrm{p} \leq 0,05)$ do tratamento constituído de $80 \%$ de substrato comercial e $20 \%$ de vermicomposto (Tabela 2). Em estudos 
realizados por Antunes et al. (2016), também foi verificada maior massa seca da parte aérea de mudas de Acacia mearnsii com adição de vermicompostos de resíduos de esterco bovino, bem como com a mistura de vermicomposto de esterco bovino e resíduos de alimentos. Conforme May \& Maranho (2007), altas concentrações de vermicomposto, na composição de substratos, induzem redução no tamanho e no número de folhas, e, por conseguinte, menor massa seca da parte aérea. Os resultados obtidos neste trabalho corroboram com os de May \& Maranho (2007), pois, na Tabela 2, pode-se observar que doses superiores a $20 \%$ de vermicomposto na composição do substrato induziram menor massa seca da parte aérea das mudas de P. elliottii.

Observam-se maior massa seca e maior área superficial específica radicular das mudas de P. elliottii com a utilização de $100 \%$ de substrato comercial, diferindo significativamente $(\mathrm{p} \leq 0,05)$ dos demais tratamentos (Tabela 2). Estes resultados são semelhantes aos de Caldeira et al. (2003), que também observaram redução na produção de massa seca radicular em mudas de P. elliottii com o aumento da proporção de vermicomposto produzido a partir de esterco bovino. Dessa forma, parece que doses elevadas de vermicomposto reduzem a massa seca das raízes das mudas de P. elliottii e isso pode estar relacionado à elevada quantidade de nutrientes disponíveis no substrato contendo proporções de vermicomposto, pois, conforme relatado por Zhang \& Forde (2000), a maior disponibilidade de nutrientes no substrato pode desestimular a expansão do sistema radicular.

$\mathrm{O}$ E. grandis apresentou menor relação H/DC no tratamento $60 \%$ de resíduo de carne com adição de $40 \%$ de esterco bovino, não diferindo $(\mathrm{p} \leq 0,05)$ do tratamento com $40 \%$ de resíduo de carne mais $60 \%$ de esterco bovino (Tabela 3). Diversamente, para o P. elliottii, a menor relação H/DC foi obtida no substrato contendo $80 \%$ de substrato comercial mais $20 \%$ de vermicomposto, diferindo estatisticamente apenas do substrato composto por $80 \%$ de resíduo de carne mais $20 \%$ de esterco bovino (Tabela 3). De acordo com Scremin-Dias et al. (2006), o diâmetro do colo espesso repercute em menor relação H/DC e quanto menor for esta relação, maior será a resistência das mudas ao plantio no campo. No entanto, neste trabalho, a menor relação H/DC não corresponde aos tratamentos nos quais se obteve maior crescimento nos parâmetros morfológicos avaliados (Tabela 2). Dessa forma, Fonseca et al. (2002) afirmaram que as relações que expressam a qualidade das mudas não devem ser utilizadas isoladamente, destacando a possibilidade de ocorrer um equívoco, se for escolhido apenas um índice como análise do padrão de qualidade.

Os tratamentos $100 \%$ de substrato comercial, substrato comercial com adição de vermicomposto e o resíduo de carne com adição de esterco bovino apresentaram maior valor do IQD para as mudas de E. grandis, diferindo significativamente $(\mathrm{p} \leq 0,05)$ do tratamento $100 \%$ resíduo de carne (Tabela 3 ). Steffen et al. (2011), em estudos com E. grandis e C. citriodora, encontraram IQD de 0,17 e 0,16 , respectivamente, aos 100 dias de avaliação. Kratz et al. (2013), trabalhando com E. benthamii, em diferentes substratos, obtiveram, em 90 dias, média do IQD de 0,17 . Porém, Gomes et al. (2002), também avaliando a qualidade das mudas E. grandis, encontraram, com 120 dias de avaliação, IQD médio de 0,032. Dessa forma, os valores encontrados no presente trabalho são intermediários aos encontrados na literatura em diferentes tipos de substrato e verifica-se que as proporções de substratos resultaram em IQD semelhantes à utilização de $100 \%$ substrato comercial (Tabela 3).

O P. elliottii revelou maior IQD com a utilização de $100 \%$ de substrato comercial, não diferindo estatisticamente $(\mathrm{p} \leq 0,05)$ do tratamento com $80 \%$ de substrato comercial e $20 \%$ de vermicomposto (Tabela 3). Hunt (1990) propôs que um valor de 0,20 seria um bom indicador de qualidade de mudas de Pseudotsuga menziesii e Picea abies, mas quanto maior for o valor de IQD, melhor será o padrão de qualidade das mudas. Nesse caso, apenas o tratamento com $100 \%$ de substrato comercial possibilitou um IQD de 0,22 para as mudas de P. elliottii.

Os tratamentos que apresentaram os maiores índices de qualidade de Dickson (Tabela 3) foram semelhantes aos tratamentos que proporcionaram médias significativamente superiores nas variáveis morfológicas (Tabela 2). Em estudos sobre a relação das variáveis de crescimento e o IQD, Binotto et al. (2010) concluíram que este índice é eficiente para indicar qualidade de mudas de E. grandis e P. elliottii, pois foi altamente correlacionado com as variáveis morfológicas das espécies. O IQD é considerado um dos melhores índices de qualidade de mudas por 
Tabela 3. Relação altura/diâmetro do colo (H/DC) e índice de qualidade de Dickson (IQD) das mudas de Eucalyptus grandis e Pinus elliottii produzidas em diferentes proporções de substratos orgânicos vermicompostados.

Table 3. Rationship height/stem diameter (H/SD) and Dickson quality index (DQI) of Eucalyptus grandis and Pinus elliottii seedlings produced in different proportions of organic substrates vermicomposted.

\begin{tabular}{|ccc|}
\hline & H/DC & IQD \\
\hline RC 100\%:EB 0\% & $7,29 \mathrm{a}^{*}$ & $0,01 \mathrm{~b}$ \\
\cline { 2 - 3 } RC 80\%:EB 20\% & $4,94 \mathrm{~b}$ & $0,09 \mathrm{a}$ \\
\hline RC 60\%:EB 40\% & $3,47 \mathrm{c}$ & $0,12 \mathrm{a}$ \\
\hline RC 40\%:EB 60\% & $4,14 \mathrm{bc}$ & $0,10 \mathrm{a}$ \\
\hline SC 100\%:VC 0\% & $7,38 \mathrm{a}$ & $0,10 \mathrm{a}$ \\
\hline SC 80\%:VC 20\% & $7,27 \mathrm{a}$ & $0,12 \mathrm{a}$ \\
\hline SC 60\%:VC 40\% & $8,27 \mathrm{a}$ & $0,10 \mathrm{a}$ \\
\hline SC 40\%:VC 60\% & $8,07 \mathrm{a}$ & $0,09 \mathrm{a}$ \\
\hline CV \% & 6,74 & 1,02 \\
\hline RC 100\%:EB 0\% & $4,64 \mathrm{ab}$ & $0,06 \mathrm{c}$ \\
\hline RC 80\%:EB 20\% & $6,09 \mathrm{a}$ & $0,05 \mathrm{c}$ \\
\hline RC 60\%:EB 40\% & $5,38 \mathrm{ab}$ & $0,08 \mathrm{c}$ \\
\hline RC 40\%:EB 60\% & $5,19 \mathrm{ab}$ & $0,07 \mathrm{c}$ \\
\hline SC 100\%: VC 0\% & $4,65 \mathrm{ab}$ & $0,22 \mathrm{a}$ \\
\hline SC 80\%:VC 20\% & $4,39 \mathrm{~b}$ & $0,17 \mathrm{ab}$ \\
\hline SC 60\%:VC 40\% & $4,92 \mathrm{ab}$ & $0,11 \mathrm{bc}$ \\
\hline SC 40\%:VC 60\% & $4,77 \mathrm{ab}$ & $0,10 \mathrm{bc}$ \\
\hline CV \% & 8,63 & 2,69 \\
\hline
\end{tabular}

$\mathrm{RC}=$ resíduo de carne; $\mathrm{EB}=$ esterco bovino; $\mathrm{VC}=$ vermicomposto $(\mathrm{RC} 40 \%: \mathrm{EB} 60 \%) ; \mathrm{SC}=$ substrato comercial. ${ }^{*}$ Médias seguidas de mesma letra na coluna não diferiram estatisticamente a $5 \%$ de probabilidade de erro pelo teste de Tukey.

considerar no cálculo a robustez e o equilíbrio da distribuição da biomassa das mudas, ponderando os resultados de vários atributos importantes em um único índice (Fonseca et al., 2002).

Os tratamentos $100 \%$ de resíduo de carne e resíduo de carne com adição de esterco bovino apresentaram menor eficiência relativa em relação ao substrato comercial, para a altura e o diâmetro do colo das mudas de E. grandis, induzindo redução de mais de $60 \%$ nessas variáveis (Figura 1A e 1B). Contudo, nos tratamentos contendo proporções de substrato comercial e vermicomposto, a altura das mudas de E. grandis foi $16 \%, 21,3 \%$ e $14,1 \%$ maiores com a adição de $20 \%, 40 \%$ e 60\% de vermicomposto, respectivamente (Figura 1A). Estes mesmos tratamentos também favoreceram a expansão do diâmetro do colo em 17,8\%, 8,2\% e 4,3\%, quando comparados ao substrato comercial (Figura 1B).

A eficiência relativa para as mudas de P. elliottii revelou que todos os substratos vermicompostados possibilitaram altura e diâmetro do colo inferior ao substrato comercial, sendo que, no tratamento 100\% resíduo de carne, as mudas de P. elliottii apresentam altura e diâmetro do colo $37,3 \%$ e $37,1 \%$ menor que a utilização de $100 \%$ de substrato comercial, respectivamente (Figura 1C e 1D). Jabur \& Martins (2002) relataram que a escolha do substrato é de fundamental importância, pois é neste que o sistema radicular irá se desenvolver, determinando o crescimento da parte aérea da muda. Deve-se, dessa forma, ter cuidado na escolha do substrato e nas suas proporções para a produção de mudas florestais. Nesse caso, parece que os tratamentos testados, com base em vermicomposto, não foram benéficos para o crescimento do $P$. elliottii.

Os resultados apresentados evidenciaram diferentes respostas de crescimento do E. grandis e do P. elliottii em relação à adição de vermicomposto na formulação de substratos. Scremin-Dias et al. (2006) afirmam que espécies de rápido crescimento demandam maiores quantidades de nutrientes e, consequentemente, apresentam maiores respostas à nutrição. Isso pode justificar a variação no crescimento das espécies do presente trabalho em relação à utilização de um mesmo tratamento (substrato), pois o pinus tem desenvolvimento mais lento, quando comparado ao eucalipto. 

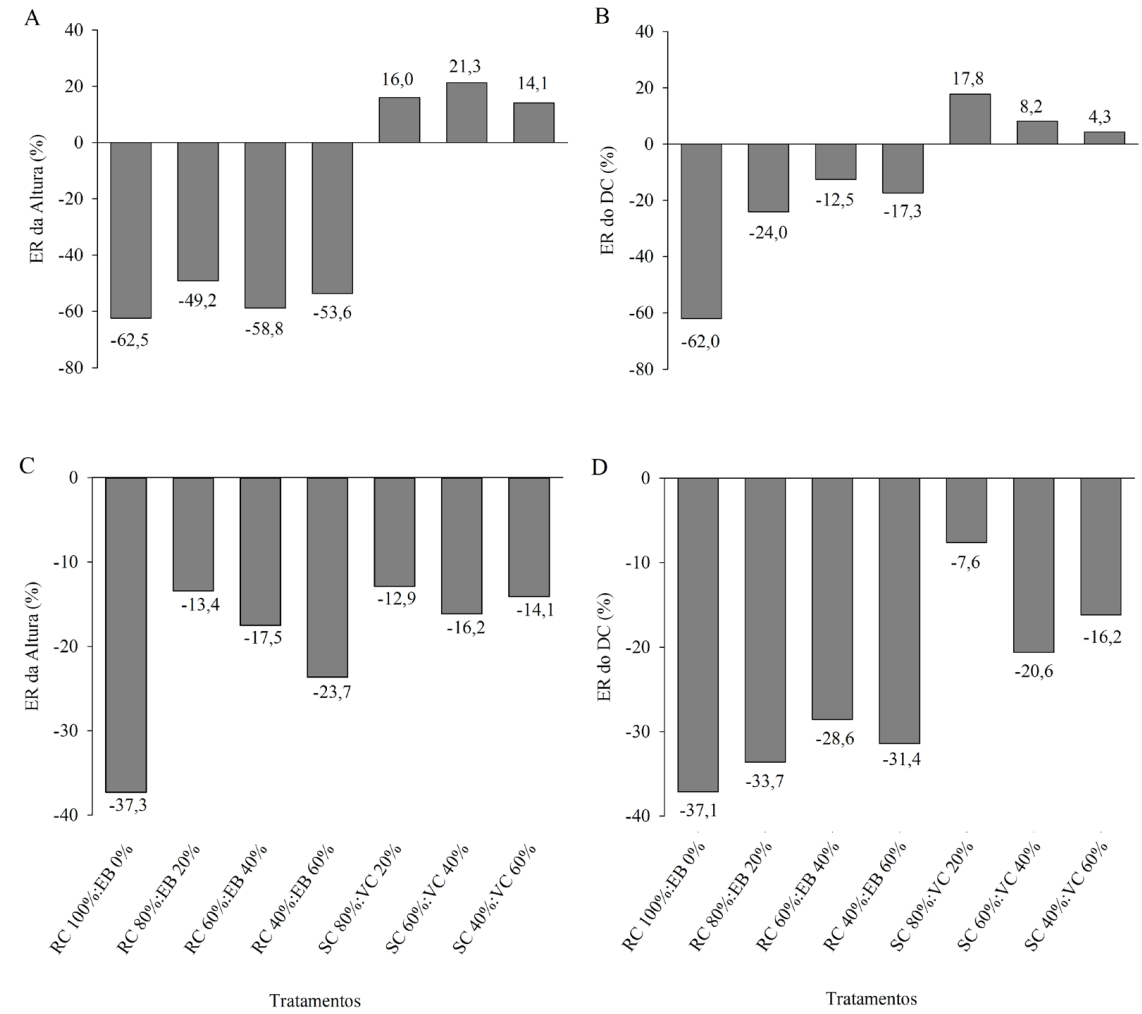

Figura 1. Eficiência relativa (ER) dos substratos em relação ao substrato comercial para a altura e o diâmetro do colo (DC) das mudas de Eucalyptus grandis (A, B) e Pinus elliottii (C, D), submetidas a diferentes proporções de substratos orgânicos vermicompostados. Onde: $\mathrm{RC}=$ resíduo de carne; $\mathrm{EB}=$ esterco bovino; $\mathrm{VC}=$ vermicomposto (RC 40\%:EB 60\%); SC = substrato comercial.

Figure 1. Relative efficiency (ER) of substrates in relation to commercial substrate to the height and diameter (DC) of Eucalyptus grandis (A, B) and Pinus elliottii (C, D) seedlings undergo different proportions of organic substrates vermicomposted. Where: $\mathrm{RC}=$ meat waste; $\mathrm{EB}=$ cattle manure; $\mathrm{VC}=$ vermicompost $(\mathrm{RC} 40 \%: \mathrm{EB} 60 \%) ; \mathrm{SC}=$ commercial substrate.

Mesmo que o vermicomposto seja considerado um bioestimulador do crescimento vegetal (Steffen et al., 2010), o efeito verificado nas mudas depende da matéria-prima utilizada para vermicompostagem, do nível de estabilização, da dose utilizada e da espécie vegetal (Edwards, 2004), possibilitando diferentes respostas entre estudos com espécies florestais.

\section{CONCLUSÕES}

A adição ao substrato comercial de $20 \%$ e $40 \%$ de vermicomposto, produzido com resíduo de carne suína e esterco de bovino nas proporções $40 \%$ e $60 \%$, proporciona desenvolvimento e qualidade de mudas de Eucalyptus grandis equivalentes à utilização de 100\% de substrato comercial.
As mudas de Pinus elliottii não são favorecidas pelas misturas de vermicompostagem testadas, apresentando maior desenvolvimento com $100 \%$ de substrato comercial.

\section{STATUS DA SUBMISSÃO}

Recebido: 7 jul., 2016

Aceito: 29 out., 2016

\section{AUTOR(ES) PARA CORRESPONDÊNCIA}

\section{Rudinei De Marco}

Departamento de Ciências Agronômicas e

Ambientais, Universidade Federal de Santa Maria

- UFSM, Linha 7 de Setembro, s/n, CEP 9840-000, 
Frederico Westphalen, RS, Brasil

e-mail: rudineidemarco@hotmail.com

\section{REFERÊNCIAS}

Antunes RM, Castilhos RMV, Castilho DD, Andreazza $\mathrm{R}$, Leal OA. Crescimento inicial de acácia-negra com vermicompostos de diferentes resíduos agroindustriais. Ciência Florestal 2016; 26(1): 1-9. http://dx.doi. org/10.5902/1980509821060.

Araújo SE No, Azevedo JMA, Galvão RO, Oliveira EBL, Ferreira RLF. Produção de muda orgânica de pimentão com diferentes substratos. Ciência Rural 2009; 39(5): 1408-1413. http://dx.doi.org/10.1590/S0103-84782009005000099.

Associação Brasileira de Produtores de Florestas Plantadas - ABRAF. Anuário Estatístico 2012 ano base 2011 [online]. Brasília: ABRAF; 2012 [citado em 2016 jan 17]. Disponível em: http://www.abraflor.org.br/estatisticas/ABRAF12/ ABRAF12-BR.pdf

Barros FD, Licco EA. A reciclagem de resíduos de origem animal: uma questão ambiental. Revista Nacional da Carne 2007; 31(365): 166-172.

Binotto AF, Lúcio AD, Lopes SJ. Correlations between growth variables and the Dickson quality index in forest seedlings. Cerne 2010; 16(4): 457-464. http://dx.doi. org/10.1590/S0104-77602010000400005.

Caldeira MVW, Schumacher MV, Oliveira ERV, Luciano ELP, Watzlawick F. Influência de vermicomposto na produção de mudas de Pinus elliottii Engelm. Revista Acadêmica: Ciências Agrárias e Ambientais 2003; 1(3): 47-53.

Dickson A, Leaf AL, Hosner JF. Quality appraisal of white spruce and white pine seedling stock in nurseries. Forestry Chronicle 1960; 36(1): 10-13. http://dx.doi.org/10.5558/ tfc36010-1.

Edvan RL, Carneiro MSS. Uso da digestiva bovina como adubo orgânico. Revista Brasileira de Tecnologia Aplicada nas Ciências Agrárias 2011; 4(2): 211-225.

Edwards CA. The use of earthworms in the breakdown and management of organic wastes. In: Edwards CA, editor. Earthworm ecology. Boca Raton: St. Lucie Press; 2004.

Ferreira DF. SISVAR: sistema de análise de variância. Versão 5.6. Lavras: UFLA; 2016.

Fonseca ÉP, Valéri SV, Miglioranza É, Fonseca NAN, Couto L. Padrão de qualidade de mudas de Trema micrantha (L.) Blume, produzidas sob diferentes períodos de sombreamento. Revista Árvore 2002; 26(4): 515-523. http://dx.doi.org/10.1590/S0100-67622002000400015.

Gomes JM, Couto L, Leite HG, Xavier A, Garcia SLR. Parâmetros morfológicos na avaliação da qualidade de mudas de Eucalyptus grandis. Revista Árvore 2002; 26(6): 655-664. http://dx.doi.org/10.1590/S0100-67622002000600002.
Hunt GA. Effect of styroblock design and cooper treatment on morphology of conifer seedlings. In: Proceedings of the Target Seedling Symposium, Meeting of the Western Forest Nursery Associations; 1990; Roseburg. Fort Collins: United States Departament of Agriculture, Forest Service; 1990. p. 218-222. General Technical Report RM-200.

Jabur MA, Martins ABG. Influência de substratos na formação dos porta-enxertos: limoeiro-cravo (Citrus Limonia Osbeck) e tangerineira-cleópatra (Citrus Reshni Hort. ex Tanaka) em ambiente protegido. Revista Brasileira de Fruticultura 2002; 24(2): 514-518. http://dx.doi. org/10.1590/S0100-29452002000200047.

Kratz D, Wendling I, Nogueira AC, Souza PVD. Substratos renováveis na produção de mudas de Eucalyptus benthamii. Ciência Florestal 2013; 23(4): 607-621. http://dx.doi. org/10.5902/1980509812345.

Lambers $\mathrm{H}$, Poorter H. Inherent variations in growth rate between higher plants: a search for fisiological causes and ecological consequences. Advances in Ecological Research 2004; 34: 283-362. http://dx.doi.org/10.1016/ S0065-2504(03)34004-8.

May D, Maranho LT. Organização estrutural da folha e influência do vermicomposto no crescimento de Menthapiperita L. Revista Brasileira de Biociências 2007; 5(2): 624-626.

Pardi MC, Santos IF, Souza ER, Pardi HS. Ciência, higiene e tecnologia da carne. 2. ed. Goiânia: Editora da UFG; 2006.

Sarzi I, Villas Boas RL, Silva MR, Carvalho JL. Características biométricas de mudas de Tabebula chrysotricha (standl.) formadas em diferentes substratos e soluções de fertirrigação, quando plantadas em campo. Revista Árvore 2010; 34(2): 241-249. http://dx.doi.org/10.1590/ S0100-67622010000200006.

Schumacher MV, Caldeira MVW, Oliveira ERV, Piroli EL. Influência do vermicomposto na produção de mudas de Eucalyptus grandis Hill ex Maiden. Ciência Florestal 2001; 11(2): 121-130. http://dx.doi.org/10.5902/19805098.

Scremin-Dias E, Kalife C, Menegucci ZRH, Souza PR. Manual de Produção de mudas de espécies florestais nativas. Campo Grande: Editora UFMS; 2006. Rede de Sementes do Pantanal, n. 2.

Silva RF, Eitelwein MT, Cherubin MR, Fabbris C, Weirich S, Pinheiro RR. Produção de mudas de Eucalyptus grandis em substratos orgânicos alternativos. Ciência Florestal 2014; 24(3): 609-619. http://dx.doi.org/10.5902/1980509815745.

Silva RF, Saidelles FLF, Silva AS, Bolzan JS. Influência da contaminação do solo por cobre no crescimento e qualidade de mudas de açoita-cavalo (Luehea divaricata Mart. \& Zucc.) e aroeira-vermelha (Schinus therebinthifolius Raddi). Ciência Florestal 2011; 21(1): 111-118. http:// dx.doi.org/10.5902/198050982753.

Steffen GPK, Antoniolli ZI, Steffen RB, Bellé R. Húmus de esterco bovino e casca de arroz carbonizada como substratos para a produção de mudas de boca-de-leão. Acta Zoológica Mexicana 2010; 26(2): 345-357. 
Steffen GPK, Antoniolli ZI, Steffen RB, Schiedeck G. Utilização de vermicomposto como substrato na produção de mudas de Eucalyptus grandis e Corymbia citriodora. Pesquisa Florestal Brasileira 2011; 31(66): 75-82. http:// dx.doi.org/10.4336/2011.pfb.31.66.75.

Tedesco MJ, Gianello C, Bissani CA, Bohnen H, Volkweiss SJ. Análise de solo, plantas e outros materiais. Porto Alegre: Universidade Federal do Rio Grande do Sul; 1995.

Tennant DA. Test of a modified line intersect method of estimating root lengh. Journal of Ecology 1975; 63(3): 995-1001. http://dx.doi.org/10.2307/2258617.
Vieira CR, Weber OLS, Scaramuzza JF. Influência do vermicomposto no crescimento e na nutrição de mudas de angico cascudo. Revista Biociências 2014; 20(2): 52-61.

Zhang HM, Forde BG. Regulation of arabidopsis root development by nitrate availability. Journal of Experimental Botany 2000; 51(342): 51-59. PMid:10938795. http:// dx.doi.org/10.1093/jxb/51.342.51. 